\title{
Comparison of Efficacy of Topical Ciprofloxacin Ear drops (0.6\%) Versus a Combination of Systemic with Topical Ciprofloxacin in Treating Chronically Discharging Ears
}

\section{Sarwat Hassan Syed, ${ }^{1}$ DamishArsalan, ${ }^{2}$ Ghulam Murtaza, ${ }^{3}$ Mohammad Qamar Nasir, ${ }^{4}$ Muhammad Awais Amin, ${ }^{5}$ AamerAyub Awan ${ }^{6}$}

\begin{abstract}
Objective: To compare the efficacy of topical ciprofloxacin alone, versus a combination therapy ofsystemic with topical ciprofloxacin $(0.6 \%)$ in achieving dry ears in active mucosal chronic otitis media after two weeks of treatment.

Methods: After obtaining permission from ethical committee of Hospital, an over-all of 150 patients (with 75 subjects each, divided into two groups) were included in this study.

In Group-A: Topical Ciprofloxacin ear drops (0.6\%) 3-4 drops were instilled three times a day, 8 hours apart for 2 weeks.

In Group-B: Tab Ciprofloxacin 500mg was given twice a day, 12 hours apart for 14 days along with topical Ciprofloxacin ear drops $(0.6 \%) 3$ drops were used thrice a day, 8 hours apart for 14 days.

Results: Patients ranged between 15-45 years of age. Mean age of the patients was 30.3 \pm 7.4 and 29.2 \pm 7.7 years. In group-A, there were 41 males (54.7\%) and in group-B 49 males (65.3\%). Females were $34(45.3 \%)$ in group-A and 27 (36\%) in Group-B. Mean duration of ear discharge was $5.3 \pm 1.1$ months in group-A while $5.5 \pm 1.4$ months in Group-B. We could not find any substantial variation among the two group in terms of efficacy $(p=0.249)$. Stratification with regard to age, gender and duration of ear discharge was also carried out.

Conclusion: Results of this study showed that topical ciprofloxacin ear drops $(0.6 \%)$ were equally effective as systemic ciprofloxacin combined with topical ciprofloxacin $(0.6 \%)$, for treating chronically discharging ears.
\end{abstract}

Keywords: CSOM, ciprofloxacin, nature of discharge

\section{Introduction}

$\mathrm{P}$ ersistently wet ears arerelated to an underlying presence of a permanent ear drum perforation and Chronic suppurative otitis media is one of the commonest causeof treatable hearing deficit around

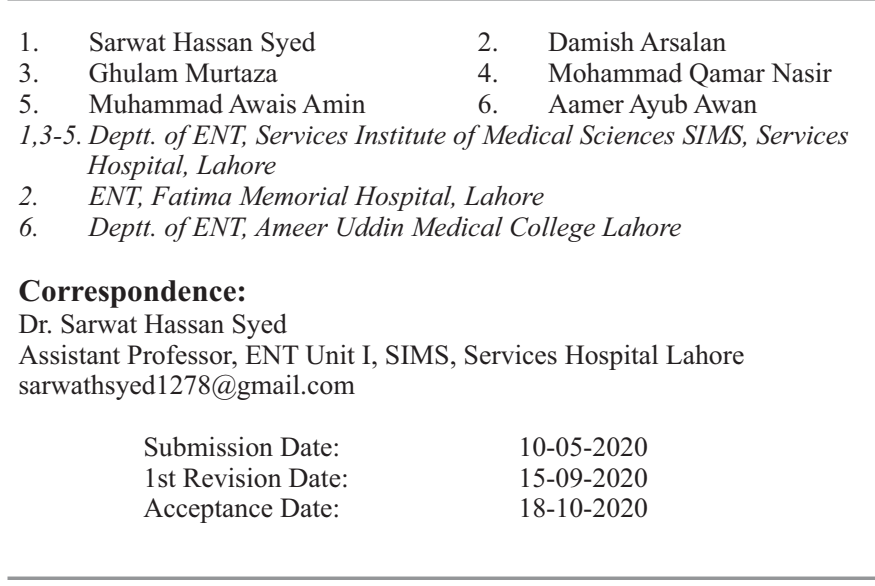

the globe, specifically in the non-affluent population with poor socio-economic conditions and limited primary health care access. Various time related definitions have been applied to CSOM; however, generally a perforation present for more than 3 months is deemed chronic. Chronic otitis media is defined as chronic inflammation of the middle ear cleft including mucosa, tympanic membrane, and ossicles. ${ }^{1}$ This type of CSOM can be further classified into active or inactive based on the incidence of ottorhoea. The inactive group includes a persistent dry perforation (failure to heal after otitis media), and retraction pockets. Active CSOM (non-cholesteatomatous) is associated with intermittent or constant ottorhoea. Most commonly isolated bacterial organisms are Staphylococcus aureus or Pseudomonas aeruginosa respectively. ${ }^{2}$ Pain is not a feature of $\mathrm{CSOM}$, as the discharge can drain freely from the 
middle ear cleft through a pre-existing or non-healing tympanic membrane perforation. The WHO definition requires that ear discharge present for more than 2 weeks' duration, manyENT clinicians contemplate the diagnosis of CSOM, despite treating it for a period wavering from 6 weeks to over 12 weeks' duration. ${ }^{3,4}$ Medical treatment should be sought for controlling the acute inflammation of CSOM.

Patients with Chronic inflammation of the middle ear cleft, present with an unremitting ear discharge through a pre-existing tympanic membrane perforation with epithelialized margins. The infection commonly begins in early childhood ${ }^{5}$ as an accompaniment of unresolved Acute Otitis Media at some point later in life. ${ }^{6}$ The infection usually begins during the first 5 years of a child's life, with a peak incidence under 2 years of age. ${ }^{7}$ The fact that how AOM progresses to CSOM is still debatable. Usually, patients with permanent ear drum perforations that continue to express mucoid aural discharge for periods ranging from 6 to 12-weeks duration8, in spite of adequate medical intervention, are categorized as CSOM cases. The WHO criteria for aural discharge in CSOM states, ottorhoea of 2 weeks duration9, but ENT clinicians are usually inclined towards adopting a lengthier duration of more than 12 weeks of active mucosal disease. ${ }^{10}$ Proliferation of sub epithelial connective tissues of middle ear mucosa and upsurge of vascularization further enhances mucus production that hamper the penetration of topical antibiotic ear drops deep into the diseased middle ear mucosa. Because of this fact, most ENT physiciansfavorthe use topical antibiotic ear drops. Topical Ear drops having Quinolones display exceptional role in eliminating Pseudomonas and are not ototoxic. ${ }^{11}$ The aim of treatment is to make the discharging ears dry and avert problems like repeated infections and hearing impairment. Out of the treatment choices, the effectiveness of using topical ciprofloxacin alone as opposed to a combination of systemic withtopical ciprofloxacin are most uncertain. In an international publication by Shia et al.; (2010) the study showed resolution of ear discharge in $70 \%$ of patients with the use of combination ciprofloxacin as compared to $50 \%$ of patients where ear discharge was resolved when topical ciprofloxacin ear drops were used for 2 weeks. ${ }^{12}$ Whereas study conducted by Renukananda et al.;(2014) showed no major difference between the two groups. ${ }^{13}$ There are no local studies available so far about this comparative research.

The objective of our research was comparison of the effectiveness of topical ciprofloxacin $0.6 \%$ ear drops alone, versus a combination of systemic ciprofloxacin $500 \mathrm{mg}$ twice daily with topical ciprofloxacin drops $0.6 \%$ thrice daily for a period of 14 days in terms of achieving dry ears in chronic otitis media. The foundation of our study was to compare the outcome of topical ciprofloxacin dropsversusa combination therapy (systemic and topical ciprofloxacin), as the afore-mentioned international studies show variations and there is controversy regarding which statement is better.

\section{Methods}

The study design opted for the present research was randomized controlled trial, conducted in the department of Otorhinolaryngology Unit-I, Services Hospital Lahore, over the period of six months, from 23-06-2016 to 22-12-2016. The sample size consisted of 150 cases, which was calculated with $80 \%$ power of test, $5 \%$ level of significance and taking expected percentage of efficacy in both groups ( 75 patients in each group respectively) i.e. $70 \%$ in combination group vs $50 \%$ in topical ciprofloxacin $(0.6 \%)$ group in achieving dry ears in active mucosal chronic otitis media after 2 weeks of treatment.

GROUP A: 75 patients were given topical ciprofloxacin $(0.6 \%) 3$ drops 8 hourly for 14 days.

Group-B: 75 patients were given Tab. Ciprofloxacin $500 \mathrm{mg}$ twice daily and topical Ciprofloxacin $(0.6 \%)$ drops 8 hours apart for 14 days.

Non probability consecutive sampling technique was used.

Patients between the age of 15 to 45 years were selected according to the following criteria which were: All patients of either gender presenting with an ear discharge of more than 2 weeks duration based on history,unilateral or bilateral COM of active mucosal variety diagnosed on Examination Under Operating Microscope (EUM). Established acute otitis media cases $(<2$ weeks' duration) with tympanic membrane perforation diagnosed on history and clinical 
examination along with inactive mucosal, active squamosal, inactive squamosal and tympanosclerosis on EUM findings and pregnant or lactating mothers were excluded from the study.

Approval from the Ethical committee of the hospital was obtained along with a written informed consent from 150 patients presenting in ENT Outpatient Department, Services Hospital, Lahore.

Written informed consent was taken from each patient at the first visit. A proforma was used for recording information of each individual in the study. Patient's biodata along with the hospital registration number was recorded in the proforma. After obtaining the informed consent, patients were arbitrarily placed into 2 groups by picking out patients' name slips from a draw box. Aural discharge was collected with a sterile ear swab from the external auditory canal. Using a sanitized aural speculum, to avoid contamination of the specimen, the sample would immediately be taken to the Pathology Lab for culture and sensitivity. The specimen was immersed in glucose growth and subsequently inoculated into Blood Agar (enriched medium) and Mckonkey Agar (differential medium) after which they were cultured for 24 hours. Culture and sensitivity of isolates was established by the Kirby-Bauer disc diffusion method. Only those patients were selected who were sensitive to ciprofloxacin.

Patients were requested to avoid water from entering into the diseased ear while showering and dry mopping of the discharge prior to putting in the ear drops was advised. The correct method of putting in the ear drops with intermittent tragal pressure for 5 minutes was advised. Patients were allowed to clean discharge from deep external meatus themselves. In the follow up visit, on 5th day of treatment, complaints were assessed. Subjective assessment was done by finding out from the patients about the absence or persistence of discharge. Objective assessment was done by otoscopic examination. If the discharge of patient failed to improve after 5th day of treatment, such patient was given systemic antibiotic other than ciprofloxacin according to culture and sensitivity of aural swab test. Results were recorded in patient proforma. Finally, patients on the line of improvement with ciprofloxacin were again evaluated for ear discharge on the final 14th day and results were added in their respective proforma.

\section{Statistical Analysis}

Statistical Data was fed into SPSS version 10 and analyzed. Descriptive statistics were calculated for both quantitative and qualitative variables. Quantitative variables e.g. age and duration of ear discharge was presented as mean \pm SD. For qualitative variables like gender and efficacy, frequency and percentage were calculated. Confounders like age, gender and duration of ear discharge was controlled through stratification. Post stratification Chi- square test will be used to compare the efficacy in two groups and $\mathrm{p} \leq$ 0.05 was considered significant.

\section{Results}

Patients age range in this study was between $15-45$ years. Mean age of the patients was $30.3 \pm 7.4$ and $29.2 \pm 7.7$ years. In group-A, there were 41 males $(54.7 \%)$ and in group-B 49 males $(65.3 \%)$. Females were $34(45.3 \%)$ in group-A and 27 (36\%) in groupB. Mean duration of ear discharge was $5.3 \pm 1.1$ months in group-A while 5.5 \pm 1.4 months in group-B. There was no substantial variation between the two study groups in terms of efficacy $(p=0.322)$. Stratifi-

Table 1: Distribution of Patients by Age

\begin{tabular}{|c|c|c|c|c|}
\hline \multirow{2}{*}{$\begin{array}{c}\text { Age } \\
\text { (Year) }\end{array}$} & \multicolumn{2}{|c|}{$\begin{array}{c}\text { Group-A } \\
\text { (Ciprofloxacin drops) }\end{array}$} & \multicolumn{2}{|c|}{$\begin{array}{c}\text { Group-B } \\
\text { (Tab. Ciprofloxacin) }\end{array}$} \\
\hline & No. & $\%$ & No. & $\%$ \\
\hline $15-30$ & 40 & 53.3 & 48 & 64.0 \\
\hline $31-45$ & 35 & 46.7 & 27 & 36.0 \\
\hline Total & 75 & 100.0 & 75 & 100.0 \\
\hline Mean \pm SD & \multicolumn{2}{|c|}{$30.3 \pm 7.4$} & \multicolumn{2}{|c|}{$29.2 \pm 7.7$} \\
\hline \multicolumn{5}{|c|}{ Table 2: Duration of Ear Discharge } \\
\hline \multirow{2}{*}{$\begin{array}{l}\text { Duration } \\
\text { (month) }\end{array}$} & \multicolumn{2}{|c|}{$\begin{array}{c}\text { Group-A } \\
\text { (Ciprofloxacin drops) }\end{array}$} & \multicolumn{2}{|c|}{$\begin{array}{c}\text { Group-B } \\
\text { (Tab. Ciprofloxacin) }\end{array}$} \\
\hline & No. & $\%$ & No. & $\%$ \\
\hline$\leq 6$ & 64 & 85.3 & 59 & 78.7 \\
\hline$>6$ & 11 & 14.7 & 16 & 21.3 \\
\hline Total & 75 & 100.0 & 75 & 100.0 \\
\hline $\operatorname{Mean} \pm$ SD & \multicolumn{2}{|c|}{$5.3 \pm 1.1$} & \multicolumn{2}{|c|}{$5.5 \pm 1.4$} \\
\hline
\end{tabular}

Table 3: Distribution of Patients by Efficacy

\begin{tabular}{ccccc}
\hline \multirow{2}{*}{ Efficacy } & \multicolumn{2}{c}{ Group-A } & \multicolumn{2}{c}{$\begin{array}{c}\text { Group-B } \\
\text { (Ciprofloxacin drops) }\end{array}$} \\
\cline { 2 - 5 } (Tab. Ciprofloxacin) \\
\cline { 2 - 5 } Yes & No. & \% & No. & $\%$ \\
No & 40 & 53.3 & 46 & 61.3 \\
Total & 35 & 46.7 & 29 & 38.7 \\
\hline
\end{tabular}

Chi Square $=0.981 \mathrm{P}$ value $=0.322$ 
cation with regard to age, gender and duration of ear discharge was also carried out.

\section{Discussion}

Chronic otitis media is defined as a chronic inflammation of the middle ear cleft including mucosa, tympanic membrane, and ossicles presenting with ear discharge through a pre-existing perforation. ${ }^{14,15}$

CSOM is the most common reason of juvenile hearing disability in developing countries. ${ }^{16}$ Correct diagnosis relies on a high index of doubt, operating micro-otoscope examination and a sensible use of screening as required. ${ }^{17,18}$

Although, its incidence has fallen in the developed world, but in developing countries, the CSOM and its sequelae still account for a major proportion of the clinical workload. Complications arise when the patient develops associated hearing disability and the social stigma of a foul smelling discharge draining from the affected ear. The morbidities associated with CSOM arises once intracranial complications ensue. ${ }^{16}$

Diagnosis depends upon reliable history taking. The main symptom is prolonged ( $>3$ months) painless otorrhea. Another common symptom is hearing impairment in the diseased ear. Adequate examination of a discharging tympanic membrane perforation will confirm the diagnosis. ${ }^{19}$

An audiogram usually shows conductive hearing loss. Bacterial cultures may not be required to ascertain the diagnosis of CSOM since $90-100 \%$ of chronically discharging ears harvest two or more segregates of both gram negative aerobes and anaerobes. Early and effective treatment based on the knowledge of causative microorganisms and their sensitivity, results in a good clinical recovery and development of complications. ${ }^{20,21}$

The most frequently isolated organism in active chronic suppurative otitis media is Pseudomonas Aeruginosa. ${ }^{22}$

Staphylococcus Aureus is the second commonest organism isolated from chronically discharging middle ears. ${ }^{22}$

Patients with CSOM respond more potently to topical rather than systemic treatment. Topical preparations can produce concentrations many times greater in the targeted tissue than those, that are not possible using systemic treatment. ${ }^{23}$

Ciprofloxacin is a second-generation FDA approved quinolone for treatment of COM in adults. Ototopical Ciprofloxacin has several advantages over Neomycin. It has the advantage of having $\mathrm{pH}$ of 6.5 , so it does not burn on administration. Its systemic absorption from topical usage is minimal, suggesting a low possibility of inducing systemic toxicity. Thus, the adverse reactions to topical Ciprofloxacin are generally mild. ${ }^{24}$

The outcomes of the current research revealed that the study groups were nearly the same in relation to age, gender and duration of ear discharge. The patients were predominantly male in both the groups. There was no majorvariation between the two study groups in terms of efficacy. Patients who received topical ciprofloxacin ear drops $(0.6 \%), 53.3 \%$ of them demonstrated dry ears within 2 weeks, while in patients taking oral ciprofloxacin $500 \mathrm{mg}$ showed efficacy in $61.3 \%$. These findings are comparable with the study of Masum and Fakir. ${ }^{6}$

\section{Conclusion}

It is therefor, concluded that the topical ciprofloxacin ear drops $(0.6 \%)$ alone, was equally effective as a combination of oral and topicalciprofloxacin in treating chronically discharging ears.

\section{Author's Contribution}

SHS: Introduction, Literature, review, Discussion

DA: Data Collection

GM,AAA: Article Reference

MQN: Statistical Analysis

MAA: Edited the article

\section{References}

1. Daniel SJ. Tropical treatment of chronic suppurativeotitis media. CurrInfect Dis Rep 2012; 14:121-7.

2. Yorgancilar E, Yildrim M, Gun R, Bakir S, Tekin R, Gocmez C, et al. Complications of chronic suppurative otitis media: a retrospective review. Eur Arch Otorhinolaryngol2012;17:23-6.

3. Afolabi OA, Salauddin AG, Ologe FE, Nwabuisi C, Nwawolo CC. Patterns of bacterial isolates in middle ear discharge with CSOM In a tertiary hospital in North Central Nigeria. Afr Health Sci2012;12:362-7. 
4. Kabir MS, Habib MA. Pattern of chronicsuppurative otitis media. Mymensingh Med J 2012; 21:270-5.

5. Jahn AF. Chronic otitis media: diagnosis and treatment. Med Clin North America 1991;75:1277-91.

6. Tos M. Sequelae of secretory otitis media and the relationship to chronic suppurative otitis media. Ann Otol Rhino Laryngol1990;99:18-19.

7. Mahoney JL. Mass management of otitis media in Zaire. Laryngoscope 1980;90:1200-8.

8. Kenna MA. Treatment of chronic suppurative otitis media. OtolaryngolClin North Am 1994;27:457-72.

9. Smith AW, Hatcher J, Mackenzie IJ, Thompson S, Bal J, Mac P, et al. Randomised control of chronic suppurative otitis media in Kenyan schoolchildren. Lancet 1996;348:1128-33.

10. Goycoolea MV, Hueb MM, Ruah C. Definitions and terminology. OtolaryngolClin North America 1991; 24:757-61.

11. Olajide TG, Fadeyi A, Segun BS. Becteriological agents of chronic discharging ears and their antibiotic sensitivity pattern in Ido-Ekiti, Nigeria. Niger Postgrad Med J 2012;19:30-5.

12. Masum S, Fakir M. Systemic antibiotics verses topical treatments for chronic discharging ears with underlying eardrum perforations. J Dhaka Med Coll 2010;19:86-90.

13. Renukananda GS. Topical verses combination in the management of discharging Chronic Suppurative Otitis Media. J Clin Diagn Res 2014; 8:KC01-KC04.

14. Acuin J. Chronic suppurative otitis media. BMJ Clin Evid 2007;2007:507.

15. Jensen RG, Koch A, Homoe P. The risk of hearing loss in a population with a high prevalence of chronic suppurative otitis media. Int $\mathrm{J}$ PediatrOtorhinolaryngol 2013;77:1530-5.

16. Vikram BK, Khaja N, Udayashankar SG, Venkatesha BK, Manjunath D. Clinico-epidemiological study of complicated and uncomplicated chronic suppurative otitis media. J LaryngolOtol2008;122:442-6.

17. Monasta L, Ronfani L, Marchetti F. Burden of disease caused by otitis media: systematic review and global estimates 2012;7:292-4.

18. Van der Veen EL, Schilder AG, van Heerbeek N. Predictors of chronic suppurative otitis media in children. Arch Otolaryngol Head Neck Surg. 2006; 132:1115-8.

19. Yorgancilar E, Yildirim M, Gun R. Complications of chronic suppurative otitis media: a retrospective review. Eur Arch Otorhinolaryngol2012;232:451-4.

20. Ahmed B, Hydri AS, Afridi AAK, Ejaz A, Farooq S, Zaidi SK. Microbiology of ear discharge in Quetta. J Coll Physicians Surg Pak. 2005;15:583-4.

21. Arshad M, Khan NU, Ali N, Afridi NM. Sensitivity and spectrum of bacterial isolates in infectious otitis externa. J Coll Physicians Surg Pak 2004; 14:146-9.

22. Jang $\mathrm{CH}$, Park SY. Emergence of ciprofloxacinresistant pseudomonas in chronic suppurative otitis media. ClinOtolaryngol Allied Sci2004;29:321-3.

23. Olagide TG, Fadeyi A, Busari SS. Bacteriological agents of chronic discharging ears and their antibiotic sensitivity pattern in ido. Niger Post grad Med J 2012;19:30-5.

24. Abes G. A Systematic Review of the Effectiveness of OfloxacinOtic Solution for the Treatment of Suppurative Otitis Media. ORL 2003;65:106-16. 